\section{A prospective description of HIV-associated multicentric Castleman disease in Malawi}

Despite high burden of Kaposi sarcoma (KS) and HIV, multicentric Castleman disease (MCD) is rarely reported in sub-Saharan Africa (SSA). A pathology series in Uganda demonstrated latency-associated nuclear antigen (LANA) and viral interleukin- 6 positivity by immunohistochemistry (IHC) in 3 of 64 reactive-appearing lymph nodes, consistent with missed MCD diagnoses, ${ }^{1}$ but this was before antiretroviral therapy (ART) scale-up when MCD may have been relatively infrequent. In South Africa, a pathology series reported many $\mathrm{HIV}^{+} \mathrm{B}$-cell lymphoproliferations without specifically mentioning $\mathrm{MCD} .^{2} \mathrm{~A}$ retrospective series from South Africa reported $35 \mathrm{MCD}$ cases during 1990-2014, with most cases in the most recent years within this time period. ${ }^{3}$

Malawi is a low-income country in SSA with $9 \%$ HIV prevalence and $66 \%$ ART coverage. ${ }^{4} \mathrm{HIV}^{+}$malignancies are common and KS accounts for one-third of all cancers. ${ }^{5}$ However, the first $\mathrm{MCD}$ cases were reported by our group in 2014-2015. ${ }^{6,7}$ This paper supplements earlier reports by describing a larger cohort of MCD patients with longer follow up, diagnosed and treated under real-world conditions in Malawi. To our knowledge, this is the first prospective cohort description of $\mathrm{HIV}^{+} \mathrm{MCD}$ from SSA, where KSassociated herpesvirus (KSHV) and HIV are endemic.

Kamuzu Central Hospital $(\mathrm{KCH})$ is the cancer referral hospital for one-half of the Malawi population (population: approx. 18 million). The KCH Lymphoma Study (clinicaltrials.gov identifier: 02835911) is a prospective observational cohort initiated in 2013 to characterize the clinicopathological spectrum of lymphoproliferative disorders in this environment in patients with and without HIV. Study procedures have been described previously. ${ }^{8}$ The study was approved by the Malawi National Health Sciences Research Committee and the University of North Carolina Biomedical Institutional Review Board, USA. Briefly, through active case finding across all hospital departments

Table 1. Baseline characteristics of patients with HIV-associated multicentric Castleman disease in Lilongwe, Malawi.

\begin{tabular}{lc}
\hline IIotal, n & 22 \\
Men, n (\%) & $14(64)$ \\
Age, years, median (range) & $41(27-57)$ \\
\hline Duration of symptoms >6 months, n (\%) & $17(77)$ \\
ART at enrollment, n (\%) & $21(95)$ \\
\hline Duration of ART, months, median (range) & $53(12-179)$ \\
CD4 count, cells/ L, median (range) & $307(2-1146)$ \\
\hline HIV RNA <2 logıcopies/ $\mu$ L, n (\%) & $15(68)$ \\
B symptoms, n (\%) & $15(68)$ \\
\hline Empiric tuberculosis treatment at enrollment, n (\%) & $8(36)$ \\
ECOG performance status, median (range) & $1(0-4)$ \\
\hline Hepatosplenomegaly, n (\%) & $19(86)$ \\
Concurrent Kaposi sarcoma, n (\%) & $3(14)$ \\
\hline Hemoglobin, g/dL, median (range) & $8.0(5.1-13.3)$ \\
Platelets, x10\%/ L, median (range) & $131(26-404)$ \\
\hline Albumin, g/dL, median (range) & $2.8(1.7-3.9)$ \\
LDH, IU/L, median (range)* & $273(133-521)$ \\
\hline
\end{tabular}

$\mathrm{N}$ : number; ART: antiretroviral treatment; CD4: cluster of differentiation 4; ECOG: Eastern Cooperative Oncology Group; LDH: lactate dehydrogenase. * $\mathrm{LDH}$ laboratory upper limit of normal is $250 \mathrm{IU} / \mathrm{L}$ and referring clinics, adults and children with newly diagnosed lymphoproliferative disorders were invited to participate after written informed consent. ${ }^{8}$ Diagnostic evaluation has also been previously described, but includes histological confirmation in real-time with immunohistochemistry (IHC) and weekly telepathology conferences using a virtual microscopy link between Lilongwe, Malawi, and Chapel Hill, USA, involving clinicians and 24 Malawian and United States (US) pathologists who reach a consensus diagnosis. ${ }^{9}$ Adults $\geq 18$ years with confirmed MCD between June 2013 and May 2018 were included in these analyses.

During most of the study, first-line treatment for MCD was etoposide $100 \mathrm{mg} / \mathrm{m}^{2}$ weekly for eight doses then biweekly for eight doses. Most patients received initial brief 5-7-day courses of steroids before starting etoposide. Second-line treatment was CVP [cyclophosphamide 750 $\mathrm{mg} / \mathrm{m}^{2}$ day 1 , vincristine $1.4 \mathrm{mg} / \mathrm{m}^{2}$ ( $\max 2 \mathrm{mg}$ ) day 1 , prednisone $60 \mathrm{mg} / \mathrm{m}^{2}$ days 1-5] every 21 days for $6-8$ cycles. This treatment protocol was initiated for several reasons: 1) availability and cost of chemotherapy agents; 2) no public sector availability nor registration for rituximab; 3) scarce data regarding MCD treatment in SSA; 4) desire to include a drug with $\mathrm{KS}$ activity to treat clinical or subclinical KS; 5) concern about multiagent chemotherapy in patients with markedly impaired performance status; 6) concern about steroids exacerbating clinical or subclinical KS; 7) use of rituximab with etoposide as a common MCD treatment in high-income countries; and 8) presence of hemophagocytic lymphohistiocytosis (HLH) in at least one case. More recently, first-line treatment was changed to CVP due to seemingly longer response durations.

After August 2016, based on frequent relapses after chemotherapy, we leveraged an ongoing phase II trial of

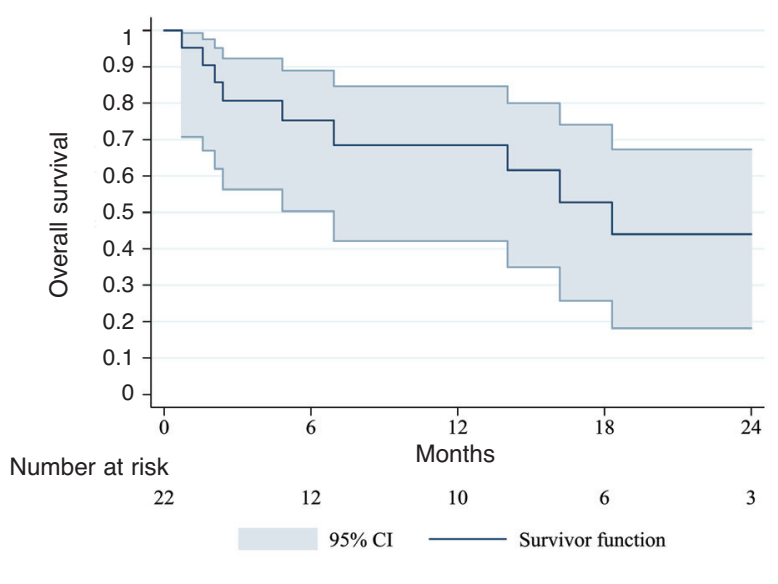

Figure 1. Histological images from a lymph node involved by multicentric Castleman disease and concomitant Kaposi sarcoma. Representative histological images are shown from a patient with concomitant (A and B) multicentric Castleman disease (MCD) and (C and D) Kaposi sarcoma (KS). Hematoxylin \& eosin stain sections (A and $C$ ) are shown along with latencyassociated nuclear antigen (LANA) immunohistochemistry (B and D). The MCD lesion is characterized by involuted germinal centers with penetrating hyalinized vessels, and prominent onion skinning of the surrounding mantle zone (A). LANA-positive plasmablasts are highlighted by immunohistochemistry (B). A separate focus of KS exhibits spindled endothelial cells with slit like spaces and numerous extravasated red blood cells $(C)$. The spindled neoplastic cells show nuclear LANA staining (D). Photomicrographs were acquired on an Olympus BX43 microscope with a 20x objective lens (UPlanFL, numerical aperture $=0.50$ ) using an Olympus DP27 camera and Olympus cellSens Standard 1.16 software. Images were sharpened and brightened using Adobe Photoshop Creative Cloud (2017.1.1 Release) without additional manipulation. 
rituximab with chemotherapy for diffuse large B-cell lymphoma in Lilongwe, Malawi, ${ }^{10}$ to petition Malawi regulatory authorities to allow compassionate use exemption for rituximab to treat relapsed MCD. For these patients, rituximab was administered at $375 \mathrm{mg} / \mathrm{m}^{2}$ weekly for four doses.

Given the lack of laboratory capacity to measure KSHV load or inflammatory markers in real time in Lilongwe, treatment response was defined as completion of therapy (16 etoposide doses, 6-8 CVP cycles, or four rituximab doses) with improved subjective systemic symptoms, improved clinical adenopathy and hepatosplenomegaly, and improved hemogloblin level, as compared with baseline. Patients were followed until death or administrative censoring on 31 May 2018. Kaplan-Meier methods were used to estimate overall survival (OS).

During the study period, $137 \mathrm{HIV}^{+}$adults with confirmed lymphoproliferative disorders were enrolled, of whom $22(16 \%)$ had MCD. All MCD patients presented with lymphadenopathy and MCD diagnosis was based on lymph node biopsy with characteristic morphology and LANA IHC positivity in all cases. An illustrative case is shown in Figure 1. MCD was approximately three times more common than $\mathrm{HIV}^{+}$Hodgkin lymphoma ( $\mathrm{n}=7,5 \%$ ) during the study period, and no HIV- or KSHV- MCD cases were diagnosed during this period. Baseline characteristics for MCD patients are shown in Table 1. Median age was 41 years (range 27-57), 14 (64\%) were male, and 8 (36\%) received empiric tuberculosis treatment before MCD diag- nosis. Twenty-one (95\%) were on ART, with median ART duration 53 months (range 12-179), median CD4 count 306 cells/ $\mu \mathrm{L}$ (range $2-1146$ ), and 15 patients $(68 \%$ ) with suppressed HIV loads. Three patients had concurrent KS, and one met diagnostic criteria for HLH. Of eight patients with baseline plasma KSHV loads tested in the US, all were positive with median level $4.1 \log _{10}$ copies/mL (range 2.7-5.2)

As of 31 May 2018, disease and vital status were known for all patients. One-year OS was 68\% (95\%CI; 42-85\%) (Figure 2). Two patients died before chemotherapy initiation. Seventeen patients were treated with first-line etoposide, of whom 11 (65\%) responded. However, eight responders experienced relapse with median time to death or next treatment of 1.2 months (range 0.2-7.8). Of 10 patients who completed second-line CVP, nine (90\%) responded, but seven experienced relapse with a median time to death or next treatment of 3.3 months (range $0.4-$ 11.9). Of these seven, five received rituximab after compassionate use exemption from Malawi regulatory authorities, and four were in remission after median follow up of 10.9 months (range 5.8-14.8). One patient developed KS worsening on rituximab requiring paclitaxel. Of nine deaths in the cohort, seven were from MCD, one from an infectious treatment-related complication, and one unrelated to MCD or treatment.

To our knowledge, this is the first well-characterized prospective cohort of $\mathrm{HIV}^{+} \mathrm{MCD}$ in SSA, a region with high HIV and KSHV burden. MCD occurred typically in
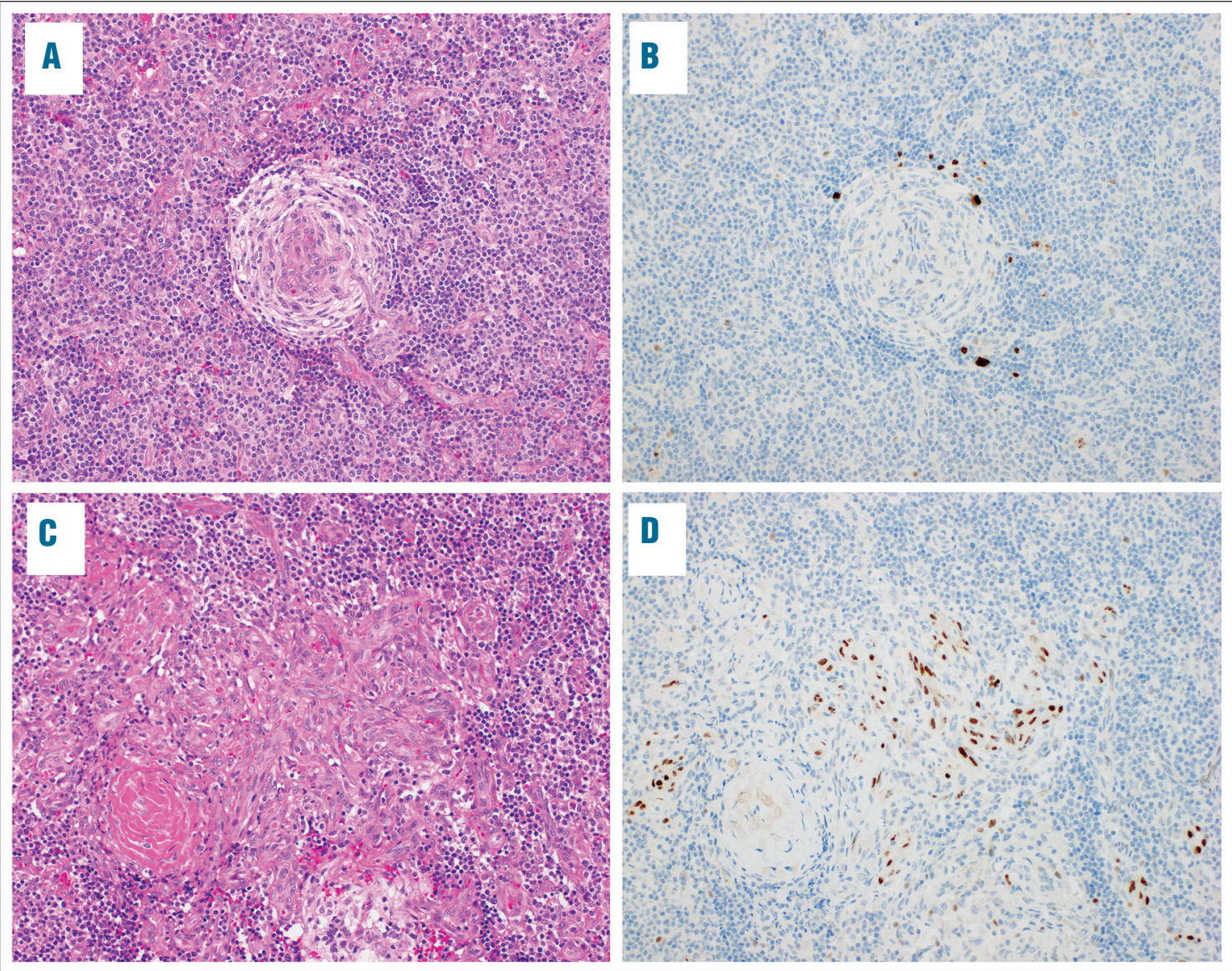

Figure 2. Overall survival for HIV-associated multicentric Castleman disease in Lilongwe, Malawi. 
patients on long-term ART. Many patients were severely ill on presentation and the mortality rate was high. Recapitulating experience in high-income countries (HICs), ${ }_{11}^{1}$ responses to chemotherapy were short-lived, and rituximab was well-tolerated with longer response durations. Unlike HICs, where MCD is highly heterogeneous with many $\mathrm{HIV}^{-}$and $\mathrm{KSHV}^{-}$cases, ${ }^{12}$ we only observed $\mathrm{HIV}^{+} \mathrm{KSHV}^{+} \mathrm{MCD}$ in our setting, which is not surprising given the high prevalence of both viruses in Malawi.

The infrequent reports of MCD in SSA are probably due to under-diagnosis; IHC is often not performed thus limiting the pathology spectrum. ${ }^{13} \mathrm{MCD}$ can mimic $\mathrm{HIV}^{+}$reactive lymphadenopathy and diagnosis is difficult without LANA IHC. In addition, the differential diagnosis of fever and lymphadenopathy in $\mathrm{HIV}^{+}$individuals is broad, ${ }^{14}$ and MCD may be missed where there is little awareness of the disease, as reflected by frequent empiric tuberculosis treatment in our patients.

Once diagnosed, MCD treatment options in SSA are limited. In our experience, chemotherapy produced transient responses with frequent rapid relapses once therapy was discontinued. After the first identification of MCD in Malawi, we were fortunate to have rituximab available for an ongoing phase II trial for diffuse large B-cell lymphoma. ${ }^{10}$ We were also fortunate to receive what may be, to our knowledge, the first ever compassionate use exemption from Malawi regulatory authorities, in order to treat relapsed MCD with rituximab. In the limited experience to date, rituximab was well-tolerated by patients with high response rates, including responses that appear more durable than those achieved after chemotherapy, even similar to resource-rich settings. ${ }^{11}$

Our experience demonstrates the value of close collaboration between SSA clinicians and pathologists. In Malawi, between 2014 and 2018, this collaboration led to the first identification of MCD and the development of a staged treatment strategy, despite severely constrained resources, which has improved outcomes compared with our initial reports. ${ }^{7} \mathrm{MCD}$ awareness in Lilongwe has increased, which is important given that MCD may increase with ART scale-up given the data in high-income countries ${ }^{15}$ and the profile of MCD patients in Malawi who typically had well-controlled HIV on long-term ART. Addressing this emerging problem will require continued investments in laboratory capacity (e.g. pathology, LANA IHC, KSHV load assays) and clinical care, including advocating for greater rituximab availability and trials to assess novel MCD agents.

In conclusion, MCD occurs relatively frequently in Malawi, and unlike HICs, is uniformly associated with HIV and KSHV. MCD presents significant diagnostic and treatment challenges in SSA. Improved awareness, laboratory capacity, and treatments are needed to address this emerging and under-recognized problem, but rapid progress is achievable even in low-income country settings through sustained multidisciplinary collaboration.

Tamiwe Tomoka, 1,2,3 Matthew S. Painschab, 2,4 Nathan D. Montgomery, ${ }^{1,2}$ Ryan Seguin, ${ }^{2}$ Maurice Mulenga, Bongani Kaimila, ${ }^{2}$ Edwards Kasonkanji, ${ }^{2}$ Takondwa Zuze, ${ }^{2}$ Richard Nyasosela, ${ }^{2}$ Ruth Nyirenda, ${ }^{2}$ Maria Chikasema, ${ }^{2}$ Blessings Tewete, ${ }^{2}$ Asekanadziwa Mtangwanika, ${ }^{2}$ Sarah Chiyoyola, ${ }^{2}$ Fred Chimzimu, ${ }^{2}$ Coxcilly Kampani, ${ }^{2}$ Yuri Fedoriw ${ }^{1,2}$ and Satish Gopal 2, 5, ,6,

'Department of Pathology and Laboratory Medicine, University of North Carolina, Chapel Hill, NC, USA; ${ }^{2}$ Malawi Cancer Consortium and Regional Center of Research Excellence for NCDs, Lilongwe, Malawi; ${ }^{3}$ Department of Pathology, University of Malawi College of Medicine, Blantyre, Malawi; ${ }^{4}$ Department of Medicine,
University of North Carolina, Chapel Hill, NC, USA; '5epartment of Epidemiology, University of North Carolina, Chapel Hill, NC, USA and ${ }^{6}$ Department of Medicine, University of Malawi College of Medicine, Blantyre, Malawi

Funding: this work was supported by grants from the Fogarty International Center (K01TW009488) and National Cancer Institute (R21CA180815, U54CA190152, P20CA210285) to SG, the Medical Education Partnership Initiative (U2GPS001965), the Lineberger Comprehensive Cancer Center (P30CA016086), the University of North Carolina Center for AIDS Research (P30AI50410), and the AIDS Malignancy Consortium (UM1CA121947).

Acknowledgments: we are sincerely grateful to the UNC Vironomics Core (Marcia Sanders, Dirk Dittmer) for assistance with plasma viral load assays in several patients, and to the Ferrata Storti Foundation for their support.

Correspondence: TAMIWETOMOKA.ttomoka@unclilongwe.org doi:10.3324/haematol.2018.204479

Information on authorship, contributions, and financial \& other disclosures was provided by the authors and is available with the online version of this article at www. haematologica.org.

\section{References}

1. Engels EA, Mbulaiteye SM, Othieno E, et al. Kaposi sarcoma-associated herpesvirus in non-Hodgkin lymphoma and reactive lymphadenopathy in Uganda. Hum Pathol. 2007;38(2):308-314.

2. Wiggill TM, Mantina H, Willem P, Perner Y, Stevens WS. Changing pattern of lymphoma subgroups at a tertiary academic complex in a high-prevalence HIV setting: a South African perspective. J Acquir Immune Defic Syndr. 2011;56(5):460-466.

3. Patel M, Philip V, Lakha A, et al. (2015). Multicentric Castleman's Disease. In: Metodiev K, ed. Immunopathology and Immunomodulation. IntechOpen. 2015;247-259. http://dx.doi.org/10.5772/ 61709. Accessed 31 May 2018.

4. UNAIDS. Malawi progress report for 2016. http://www.unaids.org/ en/regionscountries/countries/malawi. Accessed 31 May 2018

5. Msyamboza KP, Dzamalala C, Mdokwe C, et al. Burden of cancer in Malawi; common types, incidence and trends: national population-based cancer registry. BMC Res Notes. 2012;5:149.

6. Gopal S, Fedoriw Y, Montgomery ND, et al. Multicentric Castleman disease in Malawi. Lancet. 2014;384(9948):1158.

7. Gopal S, Liomba NG, Montgomery ND, et al. Characteristics and survival for HIV-associated Multicentric Castleman disease in Malawi. J Int AIDS Soc. 2015;18:20122.

8. Gopal S, Fedoriw Y, Kaimila B, et al. CHOP Chemotherapy for Aggressive Non-Hodgkin Lymphoma with and without HIV in the Antiretroviral Therapy Era in Malawi. PLoS One. 2016; 11(3): 0150445

9. Montgomery ND, Liomba NG, Kampani C, et al. Accurate RealTime Diagnosis of Lymphoproliferative Disorders in Malawi Through Clinicopathologic Teleconferences: A Model for Pathology Services in Sub-Saharan Africa. Am J Clin Pathol. 2016;146(4):423430.

10. Gopal S, Kaimila B, Kasonkanji E, et al. Rituximab in Malawi: Early Results From a Phase II Clinical Trial. Paper presented at International Conference on Malignancies in HIV/AIDS. 23-24 October 2017. Bethesda, Maryland. https://www.cancer.gov/aboutnci/organization/oham/hiv-aids-research/oham-research/international-conference/icmaoi-2017.pdf. Accessed 13 August 2018.

11. Lurain K, Yarchoan R, Uldrick TS. Treatment of Kaposi Sarcoma Herpesvirus-Associated Multicentric Castleman Disease. Hematol Oncol Clin North Am. 2018;32(1):75-88.

12. Yu L, Tu M, Cortes J, et al. Clinical and pathologic characteristics of HIV- and HHV-8- negative Castleman disease. Blood. 2017; 129(12):1658-1668

13. Tomoka T, Montgomery ND, Powers E, et al. Lymphoma and pathology in Sub-Saharan Africa: Current approaches and future directions. Clin Lab Med. 2018;38(1):91-100.

14. Bogoch II, Andrews JR, Nagami EH, Rivera AM, Gandhi RT, Stone D. Clinical predictors for the aetiology of peripheral lymphadenopathy in HIV-infected adults. HIV Med. 2013;14(3):182-186.

15. Powles T, Stebbing J, Bazeos A, et al. The role of immune suppression and HHV-8 in the increasing incidence of HIV-associated multicentric Castleman's disease. Ann Oncol. 2009;20(4):775-779. 